\title{
Tipos de pasajes sobre enfermos individuales y su inserción en el todo en Epidemias II, IV y VI ${ }^{1}$
}

\author{
Alicia Esteban Santos \\ Universidad Complutense de Madrid \\ aesteban@filol.ucm.es
}

Recibido: 30 de noviembre 2010

Aceptado: 15 de diciembre de 2010

\begin{abstract}
RESUMEN
Este trabajo trata de la distinción entre los tipos de pasajes (los generales y los individuales) en Epidemias II, IV y VI, y se centra especialmente en los pasajes individuales, sobre enfermos individuales, y en sus diferentes tipos a su vez: las «historias clínicas» y los pasajes introducidos por oĩov, etc., en que el paciente es citado como ejemplo o en comparación con otro. Se observan significativas divergencias en Epid. IV y una mayor coincidencia entre Epid. II y VI.
\end{abstract}

Palabras clave: Epidemias hipocráticas: libros II, IV y VI, pasajes de tipo diverso, divergencias y paralelismos

\begin{abstract}
This paper deals with the distinction between kinds of passages (the general passages and the individual ones) in Epidemics II, IV and VI, and is specially focussed on the individual passages, about individual patients, and their different kinds also: the "clinical histories" and the passages introduced by oiov, etc., where the patient is mentioned like example or in comparison with another. Significant divergences in Epid. IV are observed, and a stronger coincidence between Epid. II and VI .
\end{abstract}

Key words: Hippocratic Epidemics: Books II, IV and VI, passages of varied kind, divergences and parallels.

Se observa una gran diferencia en lo que respecta a la composición entre este grupo de libros de Epidemias -II, IV y VI-y el de Epidemias I y III en especial². Mayor simi-

\footnotetext{
${ }^{1}$ Este trabajo pertenece al proyecto MICINN (FFI 2009-10118) «Estudios sobre el Corpus Hippocraticum y su influencia», bajo la dirección del Dr. Ignacio Rodríguez Alfageme.

${ }^{2}$ Sin embargo, en otros aspectos, de contenido, Deichgräber (19712: 169s.) señala la mayor proximidad entre el grupo de libros I y III y el de II, IV y VI, en los que domina el pronóstico, mientras que en Epid. V y VII es más fuerte el interés terapéutico. También se acercan por la abundancia de pasajes de tipo catástasis, en especial los que, esparcidos en los tres libros -II, IV y VI-, podrían constituir un solo capítulo, denominado «tos de Perinto», que algunos investigadores han atribuido incluso al mismo autor de Epidemias I y III. Cf. Grmek (1980: 219).
} 
litud existe en ciertos aspectos con el grupo de libros V y VII, el libro IV en particular, como veremos.

Los tres grupos de tratados de Epidemias constan de pasajes de carácter general y de otros con referencia a enfermos individuales ${ }^{3}$. Pero, al contrario de lo que sucede en Epid. I y III, la composición de estos tres libros -II, IV y VI- es desorganizada ${ }^{4}$, pues se entremezclan los pasajes generales y los individuales. Los primeros predominan en los libros II y VI, mientras que el libro IV está compuesto fundamentalmente de historias clínicas 5 .

También en Epid. V y VII se mezclan de manera desordenada unos y otros tipos de pasajes; pero las historias clínicas abundan más que los pasajes generales (al igual que ocurre en el libro IV del grupo ahora considerado), y, aunque son muy diversas en cuanto a su estructura, algunas de ellas presentan un desarrollo y sistematización notables. Frente a los otros dos grupos de libros, en Epid. I y III las partes generales y las individuales se encuentran separadas de manera regular y organizada, bien delimitadas: las katastáseis o constituciones, por un lado, y las historias clínicas, por otro, aunque también se intercalan en las partes generales, de manera muy breve y escueta, algunos ejemplos individuales de cuando en cuando (pero sólo en la $3^{\mathrm{a}}$ constitución, la que precisamente precede a la sucesión más numerosa de historias clínicas). Y tales historias clínicas en Epid. I y III son sistemáticas, precisas, pormenorizadas, bastante completas en general, y siguen un esquema fijo, lo que no se da en los otros dos grupos de libros, y menos aún en el de Epidemias II, IV y VI'

${ }^{3}$ Ya en trabajos anteriores habíamos hecho esta primera distinción entre los tipos de pasajes, aunque a su vez cada una de las dos categorías en contraste las subdividimos en otras. $C f$. Esteban Santos (2009: 125ss.). Otras son las dos categorías que Deichgräber $\left(1971^{2}: 24\right)$ distingue fundamentalmente: por un lado, la gran masa de material de observación -que de nuevo separa en descripciones de catástasis e historias de enfermos individuales- y, por otro lado, la gran cantidad de formulaciones generales, notas y aforismos. En cuanto a la diferenciación más tradicional, se suelen considerar tres tipos de contenidos: descripciones de la meteorología y las enfermedades en un lugar y tiempo determinados (las catástasis), los casos individuales y, en fin, proposiciones generales extraídas de la experiencia (aforismos). Así, entre otros, Robert (1975: 173), Jouanna-Grmek (2000: VIII-IX). Ya antes lo había señalado de este modo Nikitas (1968: 40) respecto a libros II, IV y VI en concreto, en su relevante y minucioso estudio sobre ellos. En todo caso, es apreciable la rica variedad de contenidos presentes en las Epidemias. Como observa Thivel (1981: 119ss.), en las Epidemias se encuentran concentrados ya todos los contenidos de la Colección hipocrática, que después se reparten en tratados especializados (p. 124). Nikitas (1968: 47-91) recoge en un análisis general los diferentes contenidos de Epid. II, IV y VI.

${ }^{4}$ Se ha dicho que eran originariamente notas quizás de varios autores unidas para un uso privado en un círculo reducido, por ejemplo, de profesores y alumnos, como comenta Langholf (1990: 137).

${ }^{5}$ Como señala García Novo (1989: 131s.), «de unos 100 apartados en los que se divide el libro II, 80 son de carácter general. En el VI la proporción es aún más alta: 140 apartados de un total de 160. En el tratado IV no pasan de una decena de párrafos».

${ }^{6}$ Las historias clínicas de Epid. II, IV y VI son simples notas, de las que se considera por lo general que no han sido aún pulidas y redactadas como ha ocurrido en el caso de las historias clínicas de Epid. I y III. Pero, como indica García Novo (1989: 133), «El mayor o menor grado de elaboración no puede convertir una historia de la que se enumeran tres o cuatro días del curso de la enfermedad en una historia en la que, como sucede en I/III, se describen los síntomas día a día en períodos de hasta tres meses. La diferencia estriba en el método de observación y en la manera de recoger los datos». En p. 134, nota 4, añade: «el interés del autor/autores de este grupo (II/IV/VI) se ha centrado en recoger todas las historias, 


\section{PASAJES GENERALES}

Respecto a los pasajes o partes generales, que aparecen en los tres grupos de tratados en contraposición con los individuales (referidos a enfermos concretos), se distinguen tres tipos diversos: unos en relato del pasado, otros en presente y otros con proyección al futuro:

En pasado: narran las afecciones que se produjeron y la situación que se dio en una época y lugar determinados. De éstos son los más interesantes las llamadas katastáseis ${ }^{7}$, constituciones, cuando además se describen pormenorizadamente las circunstancias climáticas.

En presente: consisten en la indicación de hechos de validez universal ${ }^{8}$ : enseñanzas, máximas, aforismos ${ }^{9}$. Son reflexivos.

Orientadas al futuro hay exhortaciones y recomendaciones, que a menudo conciernen al tratamiento.

De nuevo en este aspecto se observa la mayor divergencia entre el grupo de Epidemias I y III y los otros dos, puesto que en los libros I y III los pasajes generales consisten en su mayoría en el relato de hechos pasados (las catástasis), y abundan mucho menos tanto los de presente (hechos de validez universal) como los de futuro (recomendaciones terapéuticas o de otro género). De modo que en Epid. I y III se opone lo general a lo individual fundamentalmente porque lo primero se refiere a varios enfermos y lo segundo a uno solo; pero constituyendo ambos tipos un relato histórico del pasado. Mientras que, por el contrario, en los demás grupos de tratados de Epidemias la oposición que se establece tiene un matiz diferente: lo general supone -lo más a menudo- una afirmación abstracta y teórica de lo que suele y puede ocurrir, y lo individual es la constatación de un suceso auténtico, que ejemplifica así la teoría ${ }^{10}$.

En cuanto a Epid. II, IV y VI -que ahora examinamos en concreto- son muy pocos los pasajes generales de pasado frente a los numerosísimos de presente sobre todo u orientados al futuro. Así pues, los pasajes individuales se hallan insertados en un contexto de estos pasajes que principalmente indican hechos de validez universal:

no preocupándose del estilo y de la composición, sino de tener una enciclopedia de datos experimentados de los que valerse».

${ }^{7}$ Sobre katástasis, cf., por ejemplo, Langholf (1990: 169ss.).

${ }^{8}$ Como indica Robert (1975: 260): «Pour transformer une phrase de catástasis en un aphorisme, il suffit d'un changement de temps, car une phrase de catástasis décrit ce qui arrivait à une collectivité dans des circonstances données, et elle s'exprime à un temps du passé... Si on la fait passer au présent, on la transforme en verité générale, en loi».

${ }^{9}$ Langholf (1989) comenta los textos «aphoristischen» en los libros de Epidemias.

${ }^{10}$ Deichgräber $\left(1971^{2}: 36,51\right)$ hace notar que el autor de Epid. II, IV y VI (pues él considera que los tres tratados son del mismo autor) intentaba obtener, por el método de la inducción, reglas científicas válidas de su material de observación para establecer un sistema. Le sigue Nikitas (1968: 58, 50, 82s.). García Novo (2002), en un estudio de los aspectos de generalización e individualismo en el Corpus Hippocraticum, señala que no son necesariamente opuestos, sino que son consideradas ambas facetas a la vez (p. 188), y que para alcanzar la generalización los médicos necesitaban usualmente empezar con el examen de lo individual (p. 193). Manetti (1990), en demostración de que la teoría y la observación suelen interactuar, analiza un pasaje del libro II (las secciones 2 y 3 ) en que se aprecia la relación entre los tres típicos elementos de las Epidemias: historias de casos, catástasis y teoría general. 
diseminados e intercalados en un todo de carácter general predominante en los libros II y VI. Pero en el libro IV -en la situación opuesta- son los pasajes generales los que se insertan en un todo de carácter individual predominante, y es en este tratado precisamente en el que más abundan los pasajes generales de relato de hechos pasados, aunque están, en todo caso, en menor medida que los de presente; pero sin tanta desproporción.

\section{PASAJES INDIVIDUALES}

Nos vamos a limitar -en una visión general- en este primer trabajo sobre Epid. II, IV y VI a establecer diferencias entre los tipos de pasajes referentes a enfermos individuales, sin atender al análisis detallado de cada uno y de su esquema, lo que es el objeto concreto de otro estudio -más amplio y detenido- en vías de realización. Como ya señalamos en un trabajo anterior con respecto a Epid. V y VII ${ }^{11}$, se puede hacer la distinción entre las historias clínicas propiamente dichas y los pasajes de mera mención de pacientes como ejemplo o comparación.

Respecto a los libros II, IV y VI, existe grave dificultad para una clasificación en firme de todos los pasajes (en este grupo de tratados más que en los otros dos), puesto que una gran parte se encuentra en una situación intermedia, y a veces el catalogarlos en un tipo u otro puede ser muy subjetivo. Sólo unas pocas entre las que llamamos «historias clínicas» presentan un desarrollo considerable, aunque nunca llegan a ser tan extensas, completas y sistemáticas como las de Epid. I y III y algunas de Epid. V y VII. Por otro lado, muchas aparecen insertadas como ejemplos en un contexto de exposición de hechos generales, pero no todas van precedidas del introductor típico, oíov, o equivalente, de modo que las que son muy escuetas y se limitan a una simple frase con escasos datos, no resulta fácil decidirse por incluirlas en un tipo u otro (historia clínica propia o bien mención de paciente como ejemplo o comparación), mientras que alguna de las introducidas por oíov o equivalente ofrece los suficientes datos como para poder catalogarla entre las historias clínicas.

Lo único que se puede afirmar de manera objetiva es que en este grupo de libros las historias clínicas propiamente dichas son, en todo caso (incluso las pocas más desarrolladas), incompletas y sin seguir apenas un esquema fijo. Predominan las que son escuetas y son frecuentes incluso las «muy escuetas», a las que en propiedad no se debería llamar «historias clínicas», pero les doy ese nombre cuando no están introducidas por oĩov o equivalente.

Haciendo esta salvedad, hemos intentado clasificar los diversos pasajes individuales distinguiendo (apartado 1) entre los que no llevan introductor (a los que llamamos «historias clínicas», con subtipos de clasificación -aunque algo discutible y subjetiva- de acuerdo a su mayor o menor desarrollo) y los que están introducidos por oĩov o equivalente (apartado 2), ya que éstos quedan así marcados formalmente como evidentes casos de ejemplo individual de una situación general o de comparación con otro caso individual (los llamamos por ello «mención de paciente como ejemplo o

\footnotetext{
${ }^{11}$ Esteban Santos (2009: 126 y 132ss.).
} 
comparación»), siendo, además, abundantes y destacados en este grupo de libros. En un apartado 3 recogeremos, por último, las referencias diversas a otros pasajes que se encuentran en algunas de las que llamamos «historias clínicas», que indican una relación entre diferentes historias clínicas (el caso más frecuente) o también a veces entre alguna de ellas y el pasaje general que ejemplifica. Así pues, distinguimos las historias clínicas que están vinculadas a otros pasajes y que, en adición a los introducidos por oĩov o equivalente, constituyen un conjunto de pasajes individuales que se hallan conectados formalmente con el contexto, aludiendo de algún modo a lo antes expuesto, frente a los que aparecen de manera aislada, ya que en los demás casos (la mayoría) las historias clínicas comienzan directamente sin ningún tipo enlace ni de relación explícita con el resto.

\section{Historias clínicas}

Las historias clínicas, las fundamentales de entre los pasajes individuales, relatan, con mayor o menor detenimiento, el proceso de la enfermedad de cada paciente. Son las que aparecen de manera sistemática y organizada en Epid. I y III y también las únicas en el texto exclusivo de Epid. V, en el que (al contrario que en el texto exclusivo de Epid. VII y en los textos paralelos, mucho más próximos entre sí) no aparecen pasajes individuales de otro tipo ni tampoco pasajes de carácter general ${ }^{12}$. Hemos hecho de éstas un intento de clasificación tal como aparecen en Epid. II, IV y V:

Tipo 1.1.: las más desarrolladas, que -aunque siempre incompletas y poco minuciosas y sistemáticas- presentan una cierta extensión y una gran parte de los datos principales del «esquema fijo» ${ }^{13}$ : 1) Identidad del paciente. 2) Ciudad. 3) Época. 4) Causa. 5) Indicación inicial de la enfermedad. 6) Descripción de los diversos síntomas (entre los que destaca la fiebre, el dolor, etc.). 7) Altibajos. 8) Terapia. 9) El transcurrir del tiempo. 10) Desenlace. 11) Reflexiones post desenlace. También otros datos que puedan aparecer, ya más esporádicos: el domicilio, la edad del paciente, su profesión o alguna cualidad, referencias personales del propio autor, referencias a otros médicos.

Tipo 1.2.: las que llamo «escuetas», que -en una situación intermedia- son breves (entre 2 y 5 líneas), desorganizadas, poco minuciosas y con escasos datos, pues faltan muchos relevantes del esquema, aunque cuentan al menos con algunos de los esenciales (unos en unos casos y otros en otros). Apuntan más bien datos concretos referentes a la enfermedad en cuestión y pocos sobre el paciente o sobre los pormenores del proceso de su propia afección, pues a menudo ni especifican el desenlace, ni está la identificación personal del paciente, sino que se le designa con frecuencia por su dolencia misma, o a veces por alguna acción -incluso del propio médico que escribe-, o de manera totalmente anónima («un hombre», «una mujer», «un joven», «otro», etc.). Éstas son, con mucho, las historias predominantes.

${ }^{12} C f$. Esteban Santos (2009: 127).

${ }^{13}$ Las historias de Epid. I y III observamos que muestran una composición bastante uniforme y que suelen presentar una serie de datos fijos, incluso siguiendo un mismo orden y a menudo expresados mediante frases hechas o fórmulas. Es lo que llamo «esquema fijo». Cf. Esteban Santos (1994: 50-69). También en el grupo de Epid. V y VII se puede apreciar un esquema de composición común más o menos en casi todas las historias clínicas, semejante además al que encontrábamos en Epid. I y III, aunque sin duda no tan uniforme y formulario como era en estos tratados ni tan bien elaborado. 
Tipo 1.3: las que llamo «muy escuetas», que propiamente no se pueden considerar historias clínicas, porque no ofrecen apenas datos (sólo uno o dos). Intercaladas muchas de ellas en los pasajes de tipo general ejemplificándolos, o bien refiriéndose a otro caso individual en comparación con él, sólo se distinguen de los pasajes que llamo «mención de paciente como ejemplo o comparación»-y que he contabilizado en otro apartado- por el hecho de que ésos están marcados evidentemente como tales, al ir introducidos por oĩov o equivalente.

\section{En el Libro II:}

Consideramos 26 «historias clínicas» en total:

- En su sección I no aparece ninguna historia clínica propiamente, pues todos los pasajes son ejemplos (apartado 2), con más o menos datos.

- En la sección II recogemos 20; pero pocas destacan como algo más completas y organizadas (tipo 1.1). Principalmente, la historia del cap. 6 es algo más extensa, organizada y -aunque también incompleta-, con más datos de lo habitual en este grupo de libros, correspondiendo a algunos de los del «esquema fijo». También -aunque con algunas reservas- se podrían considerar en esta categoría las de cap. 7 y 17 (ésta aún con mayor duda). Por otra parte, como «muy escuetas» (tipo 1.3) se pueden clasificar la de cap. 9 (sobre tres pacientes relacionados), la de cap. 14-15, aunque éste es un texto corrupto, y una de las de cap. 23.

- En la sección III hay pocas historias -sólo 5-, pero en general más detalladas (en especial, las de cap. 4 y 11, que son de las más extensas dentro de estos tratados, con mayor precisión de datos y estructura mas «formularia», al modo de las de Epidemias I y III), catalogables todas en el tipo 1.1, y, en cualquier caso, ninguna como «muy escueta» (del tipo 1.3).

- Una sola historia en la sección IV, y ninguna ni en la V ni en la VI, pues no hay en ellas pasajes de tipo individual.

El libro II contiene, por tanto, pocas historias. Se hallan concentradas casi todas en la sección II (21) y en la III (4) y otra más en la IV, porque éste, al igual que el libro VI (con el que coincide además por el predominio de los pasajes generales y en otros aspectos, como veremos), está distribuido en secciones. Unas 7 historias se podrían considerar del tipo 1.1, más desarrolladas, y otras 3 del tipo 1.3, muy escuetas, aunque insisto en que la referencia numérica no es rigurosa, pues no es totalmente claro en muchos casos en dónde establecer el límite. Pero, de cualquier modo, la mayoría de ellas se halla en una situación intermedia, dentro del tipo «escuetas» (tipo 1.2), con pocos datos. Sin embargo, hay algunas excepciones muy notables, que se hallan sobre todo en la sección III, que, precisamente, va introducida por un capítulo muy largo (cap. 1) referente a la tos de Perinto (tema que ofrece máximo interés en este grupo de tratados $\left.^{14}\right)$, de auténtica katástasis, al modo de las de Epid. I y III: se inicia con la

${ }^{14}$ Sobre las epidemias de Perinto, $c f$. Deichgräber (19712: 22-35) y Grmek (1980), que comenta (p. 218) en concreto de este pasaje, II 3, 1, e igualmente de VI 7, 1, que contrastan por su perfección con las notas inacabadas y las exposiciones clínicas someras que los rodean, y considera que corresponden exactamente al proceder intelectual particular que da un valor excepcional a las catástasis de los Libros I y III, con respecto a los que advierte una gran similitud de estilo, composición y, sobre todo, doctrina médica, como también antes observó Deichgräber (1971²: 26 y 33). 
mención del lugar (Perinto), sigue la amplia descripción meteorológica, con especificación de las distintas estaciones del año, $\mathrm{y}$, a continuación, narra detenidamente las afecciones que se produjeron en aquel lugar, por aquella época y bajo aquellas circunstancias climáticas.

\section{En el Libro IV:}

He contabilizado 97 «historias clínicas», aunque en este tratado resulta particularmente difícil decidir el encuadrar muchas de ellas dentro de un tipo u otro (según el mayor o menor número de datos), por su gran variedad, pues son heterogéneas -más que en los otros tratados- y parecen distinguirse varios grupos de estilos diferentes y estructura poco uniforme. Además (como ya hemos indicado), algunas muy escuetas podrían considerarse más propiamente como ejemplo o comparación, aunque no vayan introducidas por oĩov o equivalente.

La mitad o algo más de la mitad (unas 50) se hallan en una situación intermedia: del tipo «escuetas» (tipo 1.2). Pero incluso las que clasificamos en el tipo 1.1 (unas 36) -porque presentan un desarrollo algo mayor o más sistemático al contener más datos del «esquema fijo»- son poco extensas y pormenorizadas en su mayoría, a excepción de muy pocas (10, como mucho): las que se encuentran en cap. $2,3,8,9,11,15,20$, 25,26 y 31 . De ellas destacaríamos a su vez como algo más completas o minuciosas las de cap. 11, 15, 26 y 31 . Pero, en todo caso, ninguna sobresale demasiado por su amplio desarrollo.

Respecto a las que llamamos «muy escuetas» (tipo 1.3), hay unas 11: 3 en cap. 1; otra en cap. 2; 2 en cap. 20; otra en cap. 23; 2 en cap. 25; otra en cap. 30, y la última en cap. 56.

\section{En el Libro VI:}

Encontramos en total 22 «historias clínicas» (todavía menos que en el libro II), aunque -como en los otros libros- no es fácil fijar un número exacto. También como en los otros dos tratados, la mayoría (13 he contabilizado) se halla en la situación intermedia, del tipo «escuetas» (tipo 1.2). Otras 4 son algo más completas y desarrolladas: en Sección IV, cap. 4; en Sección VIII, cap. 29 -aunque ésta más dudosamente-, 30 y 32. Otras 3, en fin, las encuadramos en el tipo «muy escuetas» (tipo 1.3): en Sección II, cap. 19; en Sección III, cap. 5; en Sección VIII, cap. 27. Aunque algunas de estas últimas ya he dicho que podrían considerarse más propiamente como ejemplo o comparación, pero sin introductor, y sería posible, por otro lado, añadir alguna otra historia de discutible clasificación incluida entre las «escuetas».

$\mathrm{Ni}$ en la sección I ni en la $\mathrm{V}$ aparecen historias clínicas, como tampoco ningún otro tipo de pasaje individual. Es en la última sección, la VIII, en donde se concentra el mayor número de ellas (8), de los tres tipos.

En suma, en la catalogación y tipificación de los pasajes individuales de este grupo de libros podemos observar más claramente quizás que en ningún otro que los límites entre unos tipos y otros son muy imprecisos y subjetivos: entre una mera cita de ejemplo y una historia clínica «muy escueta»; entre las historias escuetas y las que están algo más desarrolladas. Es cuestión casi siempre de la mayor o menor extensión 
y cantidad de datos, pero no de la «calidad»; es decir, de la exposición organizada, sistemática, minuciosa y completa de éstos, que no se da en grado elevado en ninguna historia clínica de los tratados en cuestión (al contrario de lo que sucede, por supuesto, en Epid. I y III, pero también en cierta medida en Epid. V y VII).

\section{Pasajes introducidos como ejemplo o comparación}

Otros pasajes individuales están introducidos por oĩov, etc., y se intercalan en los pasajes de tipo general evidentemente como ejemplo ${ }^{15}$ (tipo 2.2), o bien en comparación con otro caso individual ${ }^{16}$ (tipo 2.3), al compararse las circunstancias de un paciente con las de otro. Suelen ser muy breves y, en gran parte, el paciente es meramente nombrado. Como ya hemos indicado en el apartado anterior, hay también pasajes que no son sino puras menciones del paciente (con sólo uno o dos datos), que ejemplifican la exposición general en la que se insertan, pero sin ningún introductor, y ésos los hemos catalogado entre las «historias clínicas», dentro del tipo «muy escuetas» (1.3).

Por otro lado, a la inversa, algunos de los pasajes introducidos por oĩov se prolongan y ofrecen un número significativo de datos, de manera que constituyen en realidad una historia clínica (un caso intermedio entre historia clínica propia y mención de paciente como ejemplo o comparación: «ejemplo amplificado», tipo 2/1), que, sin embargo, hemos incluido en este apartado, a causa de su introductor.

Vamos a centrar nuestro estudio principalmente en tales pasajes de ejemplo o comparación, así como también en los otros pasajes individuales que presentan alguna expresión que indique su conexión con lo expuesto anteriormente.

\section{En el Libro II:}

Son 12 (o 11) $)^{17}$ en total: 11 (10) de ellos van introducidos por oíov $(9$ [8]) / oĩos (1)/ oĩa (1), y otro, por ö

En su sección I hay 6 (5) pasajes como ejemplos individuales dentro de la extensa exposición general (mientras que no aparece sin embargo ninguna historia clínica propiamente). Todos van introducidos por õ̃ov (oĩos el de cap. 10). De ellos, uno (el primero de cap. 7) es del tipo 2/1, lo que podríamos definir como «ejemplo amplificado» o «historia-ejemplo», pues no se limita a ser una mera mención del paciente, sino que se extiende -aunque brevemente- en ofrecer algunos datos, al modo de una historia clínica.

En la sección II aparecen 3. De éstos, 2 como ejemplos individuales dentro de la exposición general (introducidos por õ̃ov) y otro como comparación, haciendo referencia a otro caso individual, una historia clínica propiamente (en cap. 1, introducido por oĩ $\alpha$ ).

En la sección III son 3, aunque aquí hay mayor variedad en cuanto a su modo de introducción: de nuevo por medio de oíov en 2, del tipo ejemplo, siendo uno de ellos -en cap. 18- del tipo 2/1, «ejemplo amplificado», con algunos datos. El otro (al

${ }^{15}$ Nikitas (1968) trata de los casos o historias de enfermos en pp. 52ss., y, en particular, de los enfermos como ejemplo (oĩov) en pp. 55-58, y distingue en esta categoría de pasajes tres diferentes tipos.

${ }^{16}$ Nikitas (1968: 55) incluye a estos pacientes mencionados en comparación a otros en la categoría de «enfermos como ejemplo», como primer subtipo.

${ }^{17}$ Contabilizamos 12 si aceptamos la conjetura de Smith (Smith 28, 8), según la cual habría aquí el

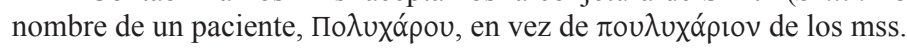


final del cap. 11) es introducido mediante ǒ $\imath 1$ koí, y está en comparación con las circunstancias de otro paciente, al que se le ha dedicado una historia clínica de las más desarrolladas y extensas.

En las secciones IV, V y VI no hay ninguno, porque en ellas no aparecen pasajes de tipo individual, a excepción de una sola historia clínica en la sección IV.

En 2 de los 12 (11) pasajes la referencia se hace a dos pacientes diferentes enlazados con koí, de modo que se menciona a 14 (13) pacientes puestos como ejemplo o comparación. De ellos sólo 2 se citan en comparación con otro paciente, y el resto son ejemplos de la exposición general, que en todos los casos consiste en un relato «en presente», afirmación teórica de lo que suele ocurrir. Por otra parte, en más de la mitad de los pasajes (en 7 de 12 [11]) se dice únicamente la identificación del paciente (su nombre o cualquier otro tipo de designación) y ningún dato más.

\section{En el Libro IV:}

Hay 14 pasajes en total, introducidos por: õ̃ov (6 [7] $)^{18} /$ oí $\alpha$ (5; entre ellos: $k \alpha i$

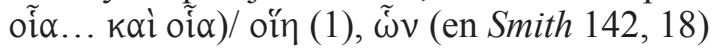

Pero en tres ocasiones son introducidas por oĩov, etc. las menciones de dos pacientes diferentes enlazados con koú, en otra con $\delta \dot{\varepsilon}$ y en otro caso se contrapone la situación

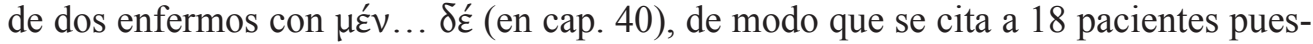
tos como ejemplo o comparación. De los pasajes, 4 se mencionan en comparación o referencia a otro paciente, y el resto (10) son ejemplos de la exposición general, que en 4 de los casos es relato de hechos pasados, frente a otros 6 casos que ejemplifican relatos «en presente», de validez universal. Por otro lado, sólo en una pequeña parte de los pasajes (en 3 de 14) se da únicamente la identificación del paciente (su nombre o cualquier otro tipo de designación), pues en la mayoría se añaden otros datos.

\section{En el Libro VI:}

Hay 14 en total, introducidos por: oĩov (11), õ̃a (1: el único ejemplo de la sección II),

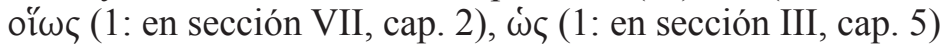

Pero en la sección VII, cap. 2, el mismo paciente es mencionado dos veces, la primera vez con la introducción oíwৎ y la segunda con õ̃ov, y en la sección III, 2 pacientes citados en cap. 5 vuelven a ser nombrados en el cap.14. Por otra parte, en la sección VIII, cap. 18, se cita a dos pacientes en yuxtaposición, con la introducción común oĩov. De modo que se mencionan 12 pacientes diferentes puestos como ejemplo o comparación. Ni en la sección I ni en la V aparecen ejemplos o comparaciones, como tampoco ningún otro tipo de pasaje individual.

No hay ningún caso en comparación o referencia a otro paciente, pues todos son ejemplos de la exposición general, que con la mayor frecuencia consiste en un relato «en presente», pero no siempre, pues en 3 casos es «de pasado» y en 1 «de futuro».

Por otra parte, en más de la mitad de los pasajes (en 8 de 14) se da únicamente la identificación del paciente (su nombre o cualquier otro tipo de designación) y ningún otro dato.

${ }^{18}$ Contabilizamos 6 si aceptamos la variante de Smith (Smith 142,18), que escoge $\tilde{\omega} v$ de la vulgata en lugar de oĩov, en cap. 48. 


\section{Otras referencias entre pasajes individuales}

Además de las menciones a pacientes individuales como ejemplo o comparación introducidas por oíov o equivalente, se encuentran referencias de otro tipo entre historias clínicas o entre alguna de éstas y otros pasajes. Entre tales referencias se encuen-

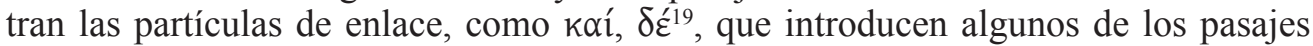
individuales indicando su conexión con lo expuesto anteriormente, ya que en los demás casos (la mayoría) las historias clínicas comienzan directamente sin ningún tipo de nexo.

\section{En el Libro II:}

En la sección II, en cap. 4, una historia clínica es introducida por koí, haciendo referencia a otro caso individual como comparación. En la sección III, cap. 18, en una

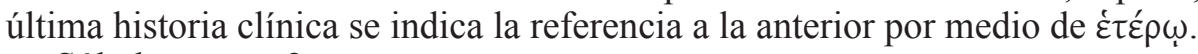

Sólo hay estos $\underline{2}$ casos.

\section{En el Libro IV:}

Hay referencias a otros en 21 casos:

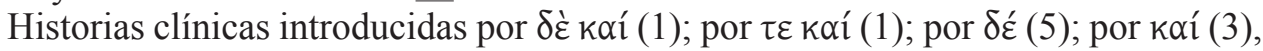
y otra más por $\mu \varepsilon ́ v$, y otra con ${ }^{\prime} \mu \alpha$.

Además:

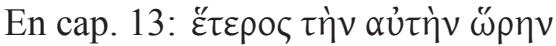

En cap. 20: $\alpha$ $\alpha \lambda$ os

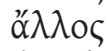

En cap. 25: $\dot{\omega} \sigma \alpha u ́ \tau \omega \varsigma$

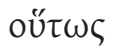

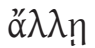

$\alpha \alpha \lambda \lambda \eta n$

$\tau \alpha u ̛$ tó

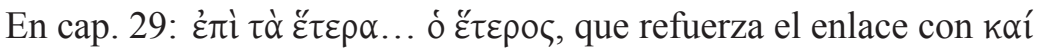

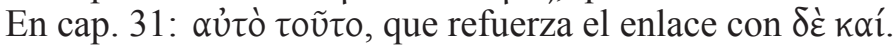

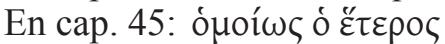

A éstos podemos añadir los casos en que se relacionan dos enfermos por su parentesco, al que se alude en cada historia independiente, como en el cap. 25: «El marido de la recién parida...» y tras la historia del hombre, la de su mujer: «La mujer de ése...». Y, principalmente, son destacables los 4 casos en que se dan dos historias entremezcladas: una intercalada en la otra ${ }^{20}$, cuya analogía el autor finalmente suele

\footnotetext{
${ }^{19}$ Acerca de la importancia del diferente uso general de tales nexos en tratados del Corpus Hippocraticum, cf. Rodríguez Alfageme (2010).

${ }^{20}$ Como también a menudo en el libro IV se intercala un pasaje de reflexión general en un caso individual, lo que es propio de Epid. IV. Nikitas (1968: 120) explica este hecho -casi exclusivo en el libro IV-de intercalar pasajes teóricos porque son casos especiales de la enfermedad en los que el autor intenta aplicar lo que ha aprendido y deducir conexiones entre sus observaciones y el conocimiento teórico. Cf. también Langholf (1990: 222): «The intercalations of general reflexions into case material, a typical feature of Epidemics IV... Observation of disease cases confirmed the theory, and the theory guides the physician's observation -un circular method».
} 
señalar con un $\alpha \dot{\mu} \mu \operatorname{có}_{\varepsilon} \rho \rho 1$. Así, en cap. 29, en cap. 31, en cap. 45 y en cap. 55. Esto no se da en los libros II y VI.

\section{En el Libro VI:}

Hay 2 historias clínicas introducidas por dè koí (una, en sección VIII, cap. 32, y otra en sección III, cap. 8, haciendo referencia ambas a otro caso individual como comparación). Una tercera historia está introducida por yó p (sección IV, cap. 13).

En la de sección VIII, cap. 32, se indica la referencia a la anterior también por me-

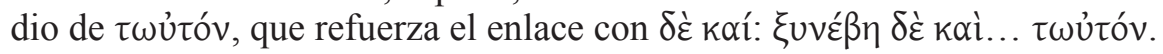

Sólo hay estos $\underline{3}$ casos.

\section{CONCLUSIONES}

No son demasiado relevantes los resultados a los que llegamos en este primer estudio -en visión muy somera- del grupo de libros de Epidemias II, IV y VI en lo que respecta a la distinción entre tipos de pasajes sobre enfermos individuales y su inserción en el conjunto del tratado respectivo. Pero nos indican al menos características coincidentes, en general, con las que se evidencian en un examen inicial y con las que han sido puestas de manifiesto por diversos investigadores desde otras perspectivas:

1.1. Diferencias entre este grupo de libros y los otros dos de Epidemias, que resultan mucho más notables en relación a Epidemias I y III, tanto en la estructura general como en la de las historias clínicas en particular, pues, por un lado, la composición de estos tres libros -II, IV y VI- es mezclada y desorganizada, y, por otro, sus historias clínicas son casi sin excepción incompletas y poco sistemáticas, incluso las más desarrolladas.

1.2. Diferencias también entre este grupo de libros y el de Epidemias V y VII, aunque a la par hay rasgos comunes (frente a Epidemias I y III): asimismo la composición de los libros V y VII es desorganizada, al entremezclarse los diversos tipos de pasajes y al presentar historias clínicas muy irregulares, de la más variada estructura. Pero las historias clínicas abundan más que los pasajes generales en Epidemias V y VII, al contrario de lo que sucede en los libros II y VI (sin embargo, no es igual en el libro IV), y, aunque muchas son escuetas y poco sistemáticas, hay algunas historias muy extensas, pormenorizadas y completas en cuanto a los datos del «esquema fijo».

1.3. En lo que respecta al tipo de los pasajes generales, la oposición de nuevo es destacada entre nuestros tratados II, IV y VI y Epidemias I y III, ya que en estos libros predominan los pasajes generales de pasado, mientras que en los libros II, IV y VI son más abundantes los de presente, al igual que en Epidemias V y VII.

2.1. Centrándonos en el tema concreto, en el examen de los tipos de pasajes individuales de Epidemias II, IV y VI, es difícil hacer una clasificación y establecer los límites y la distinción clara entre unos y otros. En esto sin duda se opone a Epidemias I y III, pero también se diferencia de los libros V y VII, en los que resulta algo más fácil distinguir las auténticas historias clínicas de los pasajes de mero ejemplo o com- 
paración ${ }^{21}$, incluso cuando éstos no sean introducidos por oĩov o equivalente, que los señalan evidentemente como tales.

2.2. Precisamente el uso de tal introductor, oĩov o equivalente, es otra característica que diferencia a los dos grupos de libros de Epidemias más afines, II, IV y VI frente a V y VII, pues en este último grupo es mucho menos frecuente el empleo de oíov o equivalente para introducir los pasajes de mera mención de pacientes como ejemplo o comparación, y se utilizan recursos más variados.

3. Por otra parte, ciñéndonos ya al grupo de libros II, IV y VI en sí, se aprecia ante todo la mayor proximidad entre los libros II y VI, frente al otro tratado, el IV:

3.1. La primera diferencia, obvia, es que en el libro IV son los pasajes individuales los predominantes en el texto, mientras que en los libros II y VI abundan más los pasajes generales.

3.2. Pero también en los otros aspectos que hemos considerado en este estudio observamos el contraste entre el libro IV y los libros II y VI ${ }^{22}$. Así, en la proporción en el uso del citado introductor, oĩov o equivalente, para indicar la conexión entre unos pasajes y otros. Tanto en el libro II como en el VI es muy elevada la proporción de pasajes individuales introducidos por oĩov, etc.: 12 con oĩov, etc., frente a $26 \sin$ introductor en el libro II, y en el libro VI, 14 con oĩov, etc., frente a 22 sin introductor; es decir, en una relación aproximada de 1:2 (mayor incluso en el libro VI). Pero en el libro IV es mucho menor: 13 pasajes introducidos por oĩov, etc., frente a unos $97 \mathrm{sin}$ introductor; es decir, en una relación aproximada de 1:7.

3.3. En cuanto a la estructura de los pasajes de ejemplo o comparación introducidos por oĩov o equivalente, asimismo hay divergencias entre el libro IV y los libros II y VI: mientras que en estos últimos predominan los pasajes en que únicamente se menciona al paciente, sin ningún otro dato, en el libro IV esto ocurre en muy pocos casos, pues en la mayoría se añaden otros datos.

3.4. Si bien proporcionalmente son pocos en el libro IV los pasajes individuales introducidos por oíov o equivalente, como hemos visto, sin embargo se emplea en este tratado, además, una amplia variedad de procedimientos de enlace y referencia en algunas historias clínicas respecto a otros pasajes, en gran contraste con relación a los libros II y VI, con el libro II en especial: aparecen referencias de algún tipo -aparte de oíov, etc.- en 21 (sin contar siquiera los casos de alusiones por parentesco) de entre las 97 historias clínicas del libro IV (21’6\%), mientras que hay sólo $2 \underline{2}$ entre las 26 del libro II (7'7\%) y $\underline{3}$ entre las 22 del libro VI (13'6\%).

\footnotetext{
${ }^{21}$ Cf. Esteban Santos (2009: 132ss.).

${ }^{22}$ La opinión probablemente más extendida es considerar relevantes las diferencias y pensar en un distinto autor para el libro IV. Así, Nikitas, Langholf, Smith (1989), que señala como única e importante preocupación en Epid. II y VI «their systematic, invasive, scientific approach» (p. 151), pues su autor intenta controlar las cosas y es extremadamente activo en su enfoque médico; mientras que en el autor del Libro IV no ve el mismo minucioso impulso teorizante, y le parece una obra independiente, con su propia orientación (p. 155ss.). Sin embargo, Deichgräber $\left(1971^{2}\right)$ advierte en los tres libros -II, IV y VI- una unidad y los cree de un mismo autor (p. 75, y también p. 74). Igualmente Robert (1975), di Benedetto (1977). Por otra parte, Grmek (1980: 217), aunque no considera un autor único, sí un único redactor de todo el conjunto, que consiste en un fichero colectivo basado en la experiencia personal de varios médicos.
} 
3.5. Una peculiaridad reiterada en el libro IV no aparece en los libros II y VI: presentar dos historias entremezcladas, con una intercalada en la otra.

3.6. De las referencias diversas contenidas en los pasajes individuales (incluyendo ahora los introducidos por oĩov o equivalente), mientras que en los libros II y VI predominan con mucho las que indican casos de ejemplificación de la exposición general, en el libro IV señalan en su inmensa mayoría menciones de pacientes en comparación a otro. Así, en el libro II hay 4 menciones de pacientes en referencia a otro (4 en un total de 14 [13] pasajes individuales en que hay referencias: $28^{\prime} 6 \%$ ). En el libro VI hay 2 menciones de pacientes en referencia a otro ( 2 en un total de 17 pasajes individuales en que hay referencias: 11 ' $8 \%$ ). Pero en el libro IV hay 28 menciones de pacientes en comparación a otro ( 28 en un total de 35 pasajes individuales en que hay referencias: $80{ }^{\prime} 0 \%$ ). La divergencia en este aspecto es, de todas maneras, lógica consecuencia del hecho de que sean predominantes los pasajes individuales en el libro IV, como también lo son quizás, en alguna medida, las conclusiones de los apartados 3.2 y 3.4

3.7. Por otra parte, cuando los pasajes individuales con tales referencias se hallan como ejemplos de la exposición general, de nuevo es relevante la disparidad del libro IV, pues en éste hacen referencia a pasajes generales «de pasado» casi tanto como «de presente», mientras que en los libros II y VI son casi exclusivamente «de presente». Como ya ha sido señalado por anteriores investigadores, el interés principal en los libros II y VI es teórico, en formulación de hechos de validez universal que son abundantemente ejemplificados con casos concretos; pero en el libro IV es más fuerte la preocupación por anotar todo lo que el médico ha ido observando efectivamente, ya sea de enfermos individuales o colectivos.

4. En la mayoría de las características ahora señaladas, el libro IV (en sus divergencias con respecto a los libros II y VI) se aproxima más que éstos al grupo de libros de Epidemias V y VII.

\section{BiBLIOGRAFÍA}

Di Benedetto, Vincenzo (1977), «Principi metodici di Epidemie II.IV.VI», en R. Joly (ed.), Corpus Hippocraticum, Actes du Colloque Hippocratique de Mons, Mons : 246-263.

Deichgrä̈er, Karl (1971²), Die Epidemien und das Corpus Hippocraticum, reimpr. BerlinNew York 1971 (Berlín 1933)

Esteban Santos, Alicia (1994), «Divergencias y paralelos entre las historias clínicas de Epidemias I y III», $C F C(G)$ 4: 47-76.

- (2009), «Incisos dentro de las historias clínicas de Epidemias V y VII: los distintos tipos de pasajes», $C F C(G)$ 19: 125-139.

García Novo, Elsa (1989), «Introducción a los libros de Epidemias. Introducción a los libros II, IV y VI de Epidemias» en A. Esteban Santos, E. García Novo, B. Cabellos, Tratados hipocráticos V. Epidemias, Madrid: 7-19 y 131ss.

- (2002), «Timelessness and generalization vs. time and individualization in the Corpus Hippocraticum and the contemporary Greek civilization» en A. Thivel, A. Zucker (edd.), Le 
normal et le pathologique dans la Collection hippocratique, Xe Colloque International Hippocratique, Nice: 179-196 (tomo I).

Grmek, Mirko D. (1980), «La description hippocratique de la "toux épidémique” de Périnthe» en M.D. Grmek (ed.), Hippocratica. Actes du Colloque hippocratique de Paris, Paris: 199-221.

Jounnna, Jacques \& Grmek, Mirko D. (2000), Hippocrate. Tome IV. $3^{e}$ Partie. Épidémies V et VII. Paris.

Langholf, Volker (1977): Syntaktische Untersuchungen zu Hippokrates-Texten. Brachylogische Syntagmen in den individuellen Krankheits-Fallbeschreibungen der hippokratischen Schriftensammlung. Wiesbaden.

- (1989), «Generalisationen und Aphorismen in den Epidemienbüchern» en G. Baader, R. Winau (edd.), Die hippokratischen Epidemien, $V^{e}$ Colloque International Hippocratique, Stuttgart : 131-142.

- (1990), Medical theories in Hippocrates. Early texts and the «Epidemics». Berlin-New York.

Manetti, Daniela \& Roselli, Amneris (1982), Epidemie. Libro sesto. Introduzione, testo critico, commento e traduzione. Firenze.

ManetTi, Daniela (1990), «Data-recording in "Epidemics" II 2-3: some considerations» en P. Potter, G. Maloney, J. Desautels (edd.), La maladie et les maladies dans la Collection hippocratique. Actes du VI Colloque International Hippocratique, Quebec: 143-158.

NikiTAS, Anastasios A. (1968), Untersuchungen zu den Epidemienbüchern II IV VI des Corpus Hippocraticum (Tesis doctoral). Hamburg.

RoBERT, Fernand (1975), «Les adresses de malades dans les Épidémies II, IV et VI» en La Collection Hippocratique et son rôle dans l'histoire de la médecine, Leiden: 173-194.

Rodríguez Alfageme, Ignacio (2010), «De locis in homine: intento de datación», $C F C(G) 20$ : $25-44$

Sмiтн, Wesley D. (1989), «Generic form in Epidemics I to VII» en G. Baader, R. Winau (edd.), Die hippokratischen Epidemien, $V^{E}$ Colloque International Hippocratique, Stuttgart : 145-158.

- (1994), Hippocrates (Vol. VII). Epidemics 2, 4-7, Cambridge (Mass.)-London.

Thivel, Antoine (1981), Cnide et Cos? Essai sur les doctrines médicales dans la Collection hippocratique. Paris. 